\section{PROCEEDINGS B}

royalsocietypublishing.org/journal/rspb

Research

Cite this article: Grecias L, Hebert F0, Alves VA, Barber I, Aubin-Horth N. 2020 Host behaviour alteration by its parasite: from brain gene expression to functional test. Proc. R. Soc. B : 20202252.

http://dx.doi.org/10.1098/rspb.2020.2252

Received: 11 September 2020

Accepted: 16 0ctober 2020

\section{Subject Category:}

Behaviour

\section{Subject Areas:}

behaviour, evolution, genomics

\section{Keywords:}

gene expression, stickleback, parasite, brain, fluoxetine, lithium

\section{Author for correspondence:}

Nadia Aubin-Horth

e-mail: nadia.aubin-horth@bio.ulaval.ca

Electronic supplementary material is available online at https://doi.org/10.6084/m9.figshare. c.5189321.

\title{
Host behaviour alteration by its parasite: from brain gene expression to functional test
}

Lucie Grecias ${ }^{1}$, Francois Olivier Hebert ${ }^{1}$, Verônica Angelica Alves ${ }^{1}$, lain Barber ${ }^{2}$ and Nadia Aubin-Horth ${ }^{1}$

\footnotetext{
${ }^{1}$ Département de Biologie et Institut de Biologie Intégrative et des Systèmes (IBIS), Université Laval, Québec, Canada

${ }^{2}$ School of Animal, Rural and Environmental Sciences, Nottingham Trent University, Nottingham, UK

(iD) IB, 0000-0003-3955-6674; NA, 0000-0002-9030-634X
}

Many parasites with complex life cycles modify their intermediate hosts' behaviour, presumably to increase transmission to their final host. The threespine stickleback (Gasterosteus aculeatus) is an intermediate host in the cestode Schistocephalus solidus life cycle, which ends in an avian host, and shows increased risky behaviours when infected. We studied brain gene expression profiles of sticklebacks infected with S. solidus to determine the proximal causes of these behavioural alterations. We show that infected fish have altered expression levels in genes involved in the inositol pathway. We thus tested the functional implication of this pathway and successfully rescued normal behaviours in infected sticklebacks using lithium exposure. We also show that exposed but uninfected fish have a distinct gene expression profile from both infected fish and control individuals, allowing us to separate gene activity related to parasite exposure from consequences of a successful infection. Finally, we find that selective serotonin reuptake inhibitor-treated sticklebacks and infected fish do not have similarly altered gene expression, despite their comparable behaviours, suggesting that the serotonin pathway is probably not the main driver of phenotypic changes in infected sticklebacks. Taken together, our results allow us to predict that if $S$. solidus directly manipulates its host, it could target the inositol pathway.

\section{Introduction}

Many parasites go through complex life cycles and as they do so alter various aspects of their host's biology, including morphology, physiology, life history and behaviour [1]. These phenotypic changes can decrease the host's fitness and have been proposed to increase the probability of completion of the parasite's life cycle [2,3], although in many cases experimental evidence is still needed [1]. Host behaviour manipulations can range from slight changes in pre-existing traits to the display of entirely novel behaviours [4]. A striking example is provided by the threespine stickleback (Gasterosteus aculeatus) and its tapeworm parasite, Schistocephalus solidus. The parasite has three hosts: a copepod, a fish (specifically the threespine stickleback), and a fish-eating bird, the definitive host [5-7]. The presence of $S$. solidus in the body cavity of its host has been reported to have multiple effects on stickleback physiology, including increased oxygen consumption [8], reduced gonad development [9] and decreased energy reserves [10]. Infected sticklebacks lose their anti-predator response and forage under the risk of predation [11-13]. They spend less time swimming within a group than healthy conspecifics [14], tend to swim away from cover, a sign of lower anxiety [15] and tend to swim close to the surface [16] even during the day, which is rarely seen in healthy conspecifics [17].

Characterizing the mechanistic basis of the interaction between the parasite and its host in the context of behavioural change requires three steps. The first one is to uncover which molecular pathways are altered in parasitized hosts. 
The second step is to use experimental manipulations to single out which molecular changes are the cause of the changes in behaviour $[15,18]$. After confirming the causal role of a molecular pathway in behaviour variation, the third step is to determine which one of these pathways, if any, is directly manipulated by the parasite [19]. Here we present data in the stickleback S. solidus host-parasite system, obtained during experiments pertaining to the two first steps. Despite a rich literature describing altered host phenotypes, there is comparatively less information on the proximate pathways involved in drastic behavioural changes in most host-parasite pairs [20,21], including in the stickleback S. solidus system [7]. We can predict that these mechanistic bases include interconnected levels of biological organization: neural circuits, neuroendocrine regulation (potentially including the serotonergic axis [22], see below), gene expression changes, and epigenetic regulation [23]. Because of the multidimensional nature of phenotypic changes in the parasitized sticklebacks, which include several types of behaviours, but also physiology [24,25] and immunity [26,27], an assumption-free wholegenome approach to characterize gene expression changes in the brain is optimal [28-30]. We can also expect that the host responds to infection [26,31] and that this will be reflected in the gene expression profiles, as found in innate and adaptive immune system genes of the stickleback's head kidney [32]. The stickleback S. solidus host-parasite system is an excellent model to study the genomic signature of parasitic infection in the host brain, i.e. a group of genes with a characteristic pattern of expression that occurs as a result of a biological process [33-35]. Sticklebacks can be experimentally infected, allowing the control of other environmental variables that could affect control and infected fish [36].

Changes in gene expression of an infected host compared to a non-infected conspecific might be functionally associated with the behavioural changes observed but could also merely be the consequence of being exposed to a parasite. An important question thus arises: do individuals exposed to a parasite that did not become infected have a similar brain expression profile to control individuals, to infected hosts, or is it unique? Because not all stickleback exposed to S. solidus become infected [7], it is possible to also study gene expression profiles of these individuals. While it has been shown that head kidney gene expression patterns do not differ between control and exposed sticklebacks [32], there are no available studies on the brain genomic signatures of exposed individuals to test these contrasting predictions.

One approach to address the question of differences in brain genomic signatures is through the study of the host's serotonergic neuroendocrinological pathway, which may be modified indirectly or directly by behaviour-altering parasites. Studies in various systems have shown changes in candidate molecules such as biogenic amines in parasitized individuals (insects [37,38], crustaceans [39], fishes [40]). In stickleback, serotonin activity is higher in S. solidus-parasitized wildcaught female sticklebacks compared to healthy females, which has been attributed to the stress of being parasitized [22]. Furthermore, experimental pharmacological manipulation of biogenic amines such as the serotonergic axis in healthy individuals results in behavioural changes typical of infected hosts (in crustaceans, [18]). In sticklebacks, selective serotonin reuptake inhibitor (SSRI)-treated non-infected sticklebacks show similar behaviours to $S$. solidus-infected individuals, with a lower tendency to school with conspecifics, and more time spent at the surface, although only in some individuals, while anti-predator response is not affected [15]. Because behavioural changes in parasitized individuals overlap in part with the ones measured in SSRI-treated individuals, they could exhibit similar activity of certain molecular pathways, which can be quantified by comparing their brain gene expression profiles.

Here, we investigated genome-wide brain gene expression patterns of sticklebacks from four treatments using RNA-seq: healthy controls, infected by $S$. solidus, exposed to a $S$. solidus parasite but not infected, and SSRI-treated. First, we analysed the transcriptome of S. solidus-infected stickleback. We predicted that they would show changes in expression of genes related to the multidimensional phenotypic changes they exhibit: behaviour, physiological systems and host response to infection. We then performed a follow-up experiment using a pharmacological manipulation, to test the behavioural effects of manipulating a candidate molecule found to be highly expressed in the brain of infected sticklebacks. Second, we included exposed individuals in which worms did not develop. We predicted that exposed fish would have a gene expression pattern mostly related to the host response to infection, which would match a subset of the expression profile of a successfully infected stickleback. Finally, we used individuals treated with the SSRI fluoxetine. We predicted that if S. solidus affects the same molecular pathways as the SSRI, we would detect a high overlap when comparing brain gene expression profiles of SSRI-treated versus infected fish.

\section{Material and methods}

\section{(a) Exposure of fish host to its parasite or selective serotonin reuptake inhibitor}

Sticklebacks from Llyn Frongoch (UK) were bred and their offspring reared in the laboratory for six months (see [15] and the electronic supplementary material). In summary, we created four treatment groups. We exposed individuals to S. solidusinfected copepods (see [15] for infection techniques) and waited three months for parasite growth. This treatment resulted in two groups: infected (fish with a parasite) and exposed fish (fish without a parasite). It was not possible to distinguish the exposed and infected individuals prior to dissection. We also exposed individuals to the SSRI fluoxetine for three days at a dose of $1 \mathrm{mg} \mathrm{l}^{-1}$ (Fluoxetine $\mathrm{HCl}, \mathrm{BML}-\mathrm{NS} 140$, Enzo Life Sciences Inc., USA), known to result in behavioural changes similar to those induced by the presence of $S$. solidus (see [15] for details). Control fish that were never exposed to a parasite were kept in the same conditions in parallel. Before fish were euthanized, they were screened for ecologically relevant behaviours, but the small sample size for infected fish $(n=3)$ prevented meaningful statistical analyses (see [15]). The parasites found in the infected sticklebacks were confirmed to be in the infective stage using their transcriptome profiles [15].

\section{(b) Gene expression quantification by RNA-seq}

Fish were euthanized following authorized protocol and dissected brains were kept in RNALater (Ambion Inc., Austin, TX, USA). We extracted total RNA from the brains of three infected, six exposed, six SSRI-treated, and six control fish (all females) using a standard Trizol reagent protocol (miRNeasy Micro kit, Qiagen) and stored at $-80^{\circ} \mathrm{C}$ after verifying concentration and quality by spectrophotometer (Nanodrop, Thermo scientifics) and a Bioanalyzer (RNA 6000 Nano Kit, Agilent Technologies Inc). We produced libraries for these 21 individuals using the 
TruSeq RNA Library Prep Kit v2 (Illumina, Inc., USA) with a unique barcode for each library. Library quality and size was assessed on a Bioanalyzer High Sensitivity DNA Assay (Agilent Technologies). The 21 complementary DNA libraries were then pooled and sequenced (Illumina HiSeq 2000). See the electronic supplementary material for details.

\section{(c) Analysis of differential gene expression}

The complete RNA-seq data preparation pipeline is available in detail in the electronic supplementary material. We used the $\mathrm{R}$ packages 'edgeR' 3.24 .3 [41] and 'limma-voom' v. 3.7 [42] to filter the dataset and determine differential gene expression. After quality control and data filtering, we used 12520 annotated transcripts and 20 of the 21 original libraries (one exposed individual was removed because of poor quality, see the electronic supplementary material, figure S1). Absolute read counts were converted into their respective 'counts per million' value and log2-transformed using the 'voom' function. Each transcript was fitted to an independent linear model using the log2(CPM) values as the response variable and the treatment as the explanatory variable. Each linear model was then analysed through limma's Bayes pipeline. We determined which genes were differentially expressed in each group (infected, exposed, SSRItreated) compared to healthy controls based on a $p$-value of $p<$ 0.005 . We did not apply a false discovery rate (FDR) correction, as it greatly reduced our dataset, with the caveat that interpretation of changes in expression of a specific gene must be done only as a preliminary result and an additional functional analysis is needed to corroborate our findings (which we did for one candidate gene, see 'Functional analysis' section and discussion). The results of statistical comparisons between control individuals and each treatment with associated fold change and $p$-value are in the electronic supplementary material, tables S1, S4 and S6. Within differentially expressed genes, we identified genes that are differentially expressed only in that specific treatment versus the control group, to define a genomic signature of that treatment group (ex: significantly more expressed in infected fish compared to controls, but not differentially expressed between exposed fish and controls, or between SSRI-treated fish and controls) $[29,35]$. These genes are marked in bold in the corresponding supplementary tables. We performed an enrichment analysis for each genomic signature separately, to test if certain biological functions were significantly over-represented. Gene ontology terms for each gene were based on the published transcriptome of Gasterosteus aculeatus. We used the Python package 'goatools' v. 0.6.5 [43] to perform Fisher's exact tests using a $p$-value of $p<0.005$ as a significance threshold.

\section{(d) Functional analysis in infected sticklebacks: pharmacological rescue of behaviour}

Several genes coding for molecules involved in the inositol pathway were found to be differentially regulated in the brain of S. solidus-infected fish (see Results section). One of them is inositol monophosphatase 1 (impa1, electronic supplementary material, table S1). This gene codes for the IMPase 1 enzyme, which is a central step in the synthesis of myo-inositol [44]. Altered inositol metabolism has been implicated in various human neuropsychiatric and neurological diseases [45]. Lithium chloride is used to diminish behavioural symptoms of these diseases, such as the manic phase symptoms observed in bipolar patients [46], which include sleeplessness, hallucinations, psychosis or paranoid rage [47]. One of the most accepted mechanisms of lithium action is the inositol depletion hypothesis [44] which suggests that lithium acts by blocking the IMPase 1 enzyme activity, leading to a depletion of inositol in the brain of treated patients $[44,45,48,49]$. Therefore, we predicted that we could rescue normal behaviour in infected stickleback by modulating the inositol pathway using lithium exposure. We measured two well-characterized behaviours in infected individuals: the tendency to swim near the surface and the response to a simulated bird strike (here the time spent frozen after an attack). For the tendency to swim near the surface test, we separated the tank into three zones vertically and water was aerated continuously. After a $5 \mathrm{~min}$ acclimation period, we tracked the fish vertical position in the tank for $5 \mathrm{~min}$. For the response to a simulated bird strike test, we also let fish acclimate in the aquarium for $5 \mathrm{~min}$. We fed the fish and once it started to eat or $5 \mathrm{~min}$ after the feeding, we performed a simulated bird strike from above using a model of a heron head. We analysed how long the fish spent frozen after the attack (not moving for at least more than $2 \mathrm{~s}$ ). Using wildcaught threespine sticklebacks from Lac Témiscouata (QC, Canada), we quantified these two behaviours in S. solidus-infected individuals before and after exposure to lithium. One group was exposed to two low doses of lithium (at $2.5 \mathrm{mM}$ and $5 \mathrm{mM}$ ), and a second group to a high dose (at $15 \mathrm{mM}$ ). Significant effects on behaviour in treated infected fish were tested for each dose using a linear mixed effects analysis of the relationship between our dependent variable (behaviour) and treatment. See the electronic supplementary material for details.

\section{Results and discussion}

\section{(a) Altered molecular pathways in the brain following an infection by Schistocephalus solidus}

There were 105 differentially expressed genes between infected and control sticklebacks: 92 upregulated and 13 downregulated in infected, with a median $\log 2$ fold change of 0.60 (range: $0.26-2.76$ ) and -0.40 (range: -0.82 to -0.28 ), respectively (electronic supplementary material, table S1). A total of 45 out of the 105 differentially expressed genes were differentially expressed only in the infected-control comparison and are thus considered as a genomic signature of infection (37 upregulated, eight downregulated), electronic supplementary material, table S1). The 45 genes forming the infected genomic signature were significantly enriched for categories associated with behaviour alterations (aromatic amino acid transport, thyroid hormone transport), host response to infection (catalase activity, oligosaccharyl transferase activity) and cellular growth (thymidylate kinase activity, dTDP biosynthetic process) (electronic supplementary material, table S2).

\section{(i) Molecular changes associated with behavioural alteration}

Aromatic amino acids include all the precursors to biogenic amines (dopamine, serotonin, and epinephrine), melatonin and thyroid hormone. Several biogenic amines are related to behaviour variation [50] and this result is in accordance with the altered serotonin metabolism found in the brain of infected fish [22]. The thyroid hormone transport function was also over-represented in genes differentially expressed in infected fish. Administration of thyroid hormone (thyroxine) in the Schistosoma mansoni host increased worm numbers and lead to the development of giant worms [51]. Thyroid hormone can also affect behaviour: treatments with thyroid hormones cause salmons to move to open water in daytime [52] and to change from a territorial phase to schooling phase during smelting [53]. Thus, increase of thyroid hormone transport might be beneficial for $S$. solidus growth and the completion of its cycle.

impal was among the upregulated genes in the brain of infected fish that is associated with behaviour (figure 1; 


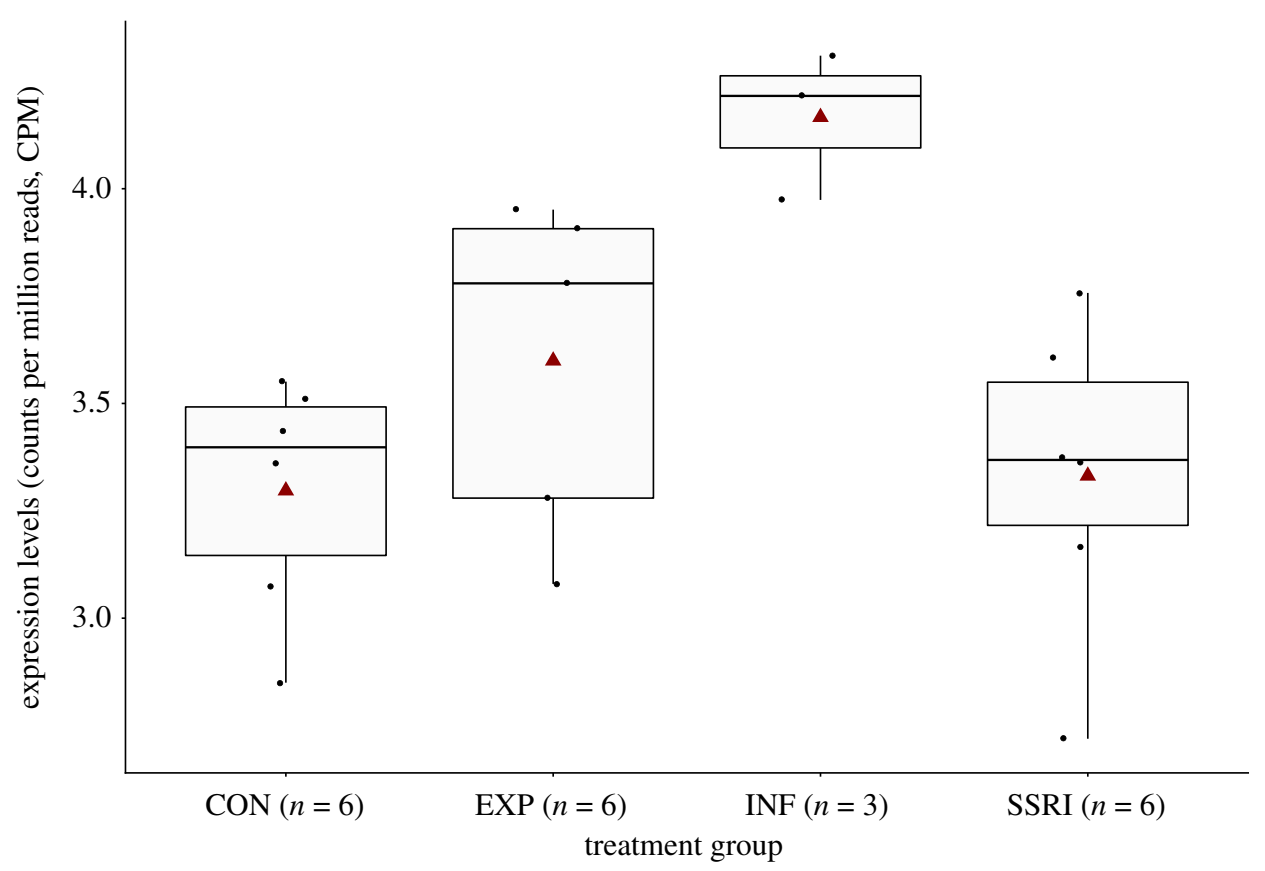

Figure 1. The IMPA 1 gene is significantly more expressed in the brain of S. solidus-infected sticklebacks compared to exposed, SSRI-treated and control fish. Box plots with median, 25th and 75th percentiles, vertical bars representing the largest value no further than 1.5 times the interquartile range, means (red triangles) and individual data (black dots). The four treatment groups are: CON, healthy controls; EXP, fish that were exposed to the parasite but did not become infected; INF, infected fish; SSRI, SSRI-treated fish; sample size in parentheses.

electronic supplementary material, table S1). This gene encodes IMPase 1, a central enzyme in the inositol pathway [48], which is implicated in a diverse range of responses in the central nervous system $[44,54]$. Alterations to this signalling pathway could be the cause of behaviour changes in infected sticklebacks. We tested the functional link between an increase in impa1 expression and behaviour by pharmacologically blocking IMPase 1 activity with lithium, which is used to treat symptoms of bipolar disorder by targeting IMPase activity $[45,49]$. We attempted to rescue two behaviours that are altered in S. solidus-infected individuals: the tendency to swim closer to the surface and the lack of response to predator attacks. Infected sticklebacks exposed to low doses of lithium chloride $(2.5$ and $5 \mathrm{mM})$ did not reduce the proportion of time they spent swimming in the upper part of the aquarium compared to the control week $(2.5 \mathrm{mM}, t$-ratio $=0.123, p=0.99, n=17 ; 5 \mathrm{mM}, t$-ratio $=-$ $0.607, p=0.82, n=17$; figure $2 a$ ). However, infected sticklebacks treated with lithium chloride at a dose of $15 \mathrm{mM}$ spent significantly less time in the top of the aquarium than before treatment (figure $2 a$; $t$-ratio $=5.69, p<0.001, n=5$ ). Infected sticklebacks treated with both low doses of lithium chloride spent almost no time frozen after a simulated bird strike, which was not significantly different from their behaviour before treatment (figure $2 b ; 2.5 \mathrm{mM}$ of lithium, $t$-ratio $=$ $0.742, p=0.74, n=17 ; 5 \mathrm{mM}$ of lithium, $t$-ratio $=-0.021, p=$ $0.99, n=17)$. However, infected fish treated with lithium chloride at a dose of $15 \mathrm{mM}$ spent significantly more time frozen after a simulated bird strike (figure $2 b$; $t$-ratio $=-2.803$, $p=0.003, n=5)$. These results suggest that lithium can block the IMPase 1 enzyme activity in the infected stickleback brain and alter their behaviour, making them respond more like healthy fish. Indeed, fish treated with high doses of lithium spent on average $7 \%$ (s.d. $=7 \%$ ) of time near the surface and $85 \mathrm{~s}$ (s.d. = $62 \mathrm{~s}$ ) frozen after a simulated bird strike, while non-infected sticklebacks studied in the same conditions in a separate study spent $6 \%($ s.d. $=6 \%)$ of time near the surface and stayed frozen $34 \mathrm{~s}$ (s.d. $=65 \mathrm{~s}$ ) (V. A. Alves and N. Aubin-Horth 2020, unpublished data). Such observations imply that alterations in the inositol pathway could be the direct cause, at least in part, of the striking behavioural alterations observed in this host-parasite model. To our knowledge, our results are one of the first examples of a pharmacological rescue of the behaviour of a host infected with a putative manipulative parasite, along with findings in the Toxoplasma-rodent system. Indeed, the risky behaviour of Toxoplasma-infected rodents towards predators has been successfully returned to cautiousness using antipsychotic drugs used to treat symptoms of schizophrenia [55].

In the stickleback S. solidus system, it remains to be tested whether the alteration of the inositol pathway is a side-effect of a host response or if it is the result of a direct manipulation by the parasite. To test these different hypotheses, a combination of approaches will be needed to gather indirect and direct evidence [19]. Such approaches include characterizing which molecules are secreted/excreted by the parasite (termed 'manipulation factors' [19]), if these manipulation factors alter the host behaviour [56], and through which molecular mechanisms in the host.

\section{(ii) Molecular changes associated with the host response}

Enrichment for certain biological functions in infected fish brains suggested that a host response to infection could be at play. The over representation of catalase activity, an important enzyme protecting the cell from oxidative damage by reactive oxygen species (ROS) [57] might be explained as a consequence of infection, because ROS production is increased in head kidney leucocytes in contact with $S$. solidus extracts in vitro [26] and appears to play an important role in stickleback defence against S. solidus [32]. However, the over representation in catalase activity might also indicate a way 

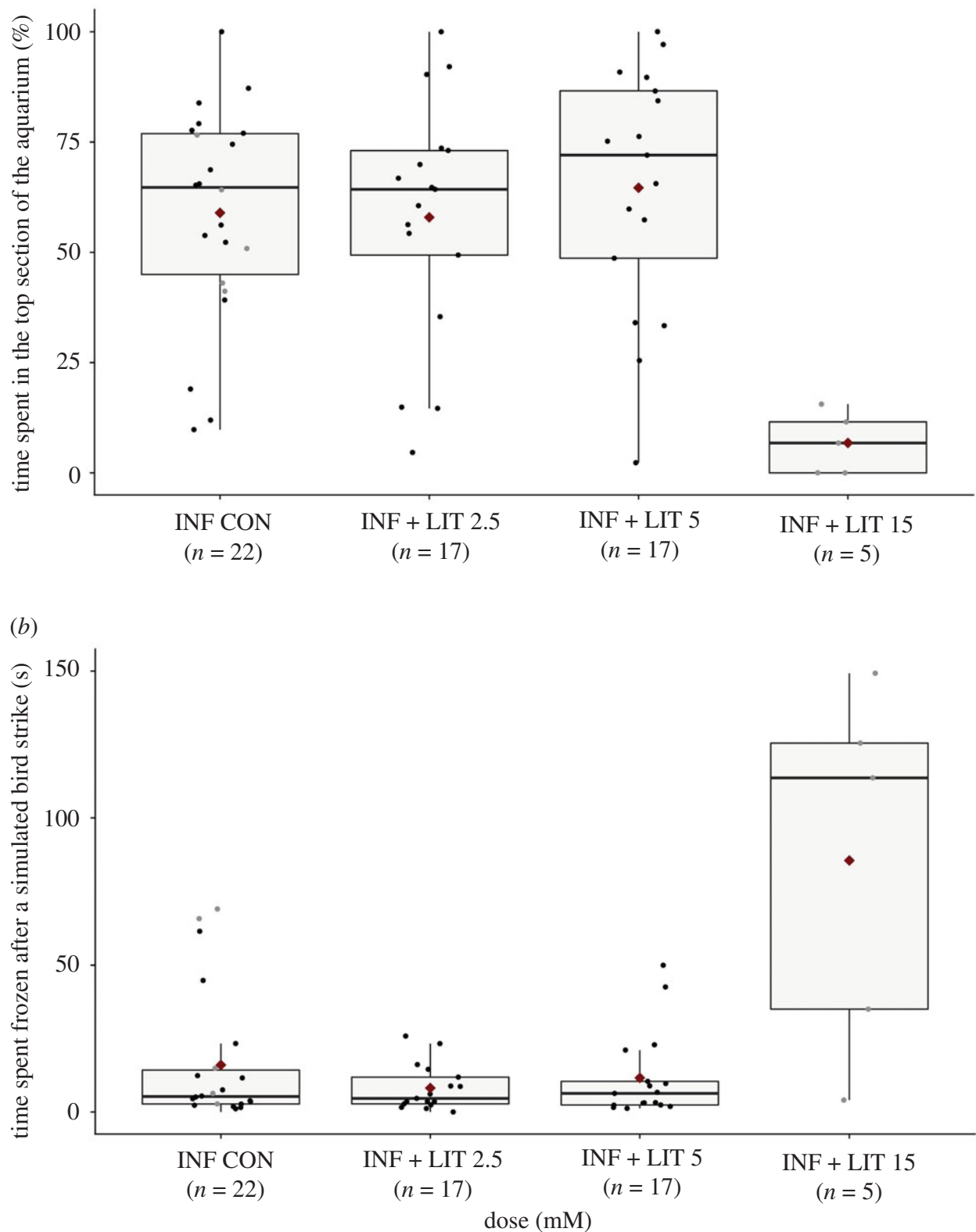

Figure 2. Lithium chloride exposure at a dose of $15 \mathrm{mM}$ rescues normal behaviours in S. solidus-infected sticklebacks. (a) Infected sticklebacks treated with lithium chloride at a dose of $15 \mathrm{mM}$ spent less time in the top of the aquarium compared to the control week ( $15 \mathrm{mM}, t$-ratio $=5.69, p=0.0007, n=5)$, while infected sticklebacks treated with lower doses of lithium chloride did not change their vertical preference after treatment $(2.5 \mathrm{mM}, t$-ratio $=0.123, p=0.99, n=17 ; 5 \mathrm{mM}$, $t$-ratio $=-0.607, p=0.82, n=17$ ). (b) Infected sticklebacks treated with lithium chloride at a dose of $15 \mathrm{mM}$ spent more time frozen after a simulated bird strike in comparison to their control week ( $15 \mathrm{mM}, t$-ratio $=-2.803, p=0.003, n=5)$, while infected sticklebacks treated with lower doses of lithium chloride did not change the time they spent frozen following a simulated bird strike after treatment $(2.5 \mathrm{mM}, t$-ratio $=0.742, p=0.74, n=17 ; 5 \mathrm{mM}, t$-ratio $=-0.021, p=0.99$, $n=17$ ). Box plots with median, 25th and 75th percentiles, vertical bars representing the largest value no further than 1.5 times the interquartile range, means (red triangles), and individual data (black dots, low lithium doses; grey dots, high lithium dose). The four treatment groups are: INF CON, infected control; INF LIT, infected fish treated with lithium chloride, with the number representing the dose in $\mathrm{mM}$, sample size in parentheses.

by which the parasite can manipulate its host in order to eliminate an oxidative stress that would otherwise compromise parasite survival [58]. This over-representation could also indicate a reaction from the host aimed at decreasing the oxidative stress caused by the parasite. Genes whose function was associated with oligosaccharyl transferase activity were also over-represented in infected fish. This transferase is implicated in post-translational modifications of proteins by glycosylation, which determines the localization and function of these proteins [59]. Again, those modifications might be a global host response to infection at the protein level that can have major effects on cellular activity, among other wideranging effects. Finally, thymidylate kinase activity and
dTDP biosynthetic process are biological functions enriched in infected fish brains that are related to cellular growth. The over-representation of the thymidylate kinase activity, an important enzyme that assists biosynthesis of mitochondrial DNA, might also be explained as a consequence of infection, as a thymidylate kinase-like gene was found upregulated in infected salmon and may be linked to the innate response to infection by a monogenean parasite [60]. An interesting feature of the stickleback $S$. solidus system is that the hypothesis of a global host response could be verified by a gene expression study in the first intermediate host of S. solidus, the copepod. Determining if the biological functions of genes differentially expressed in infected copepod mirror the 
ones found in infected fish would allow us to determine the degree of overlap in the molecular response of both hosts and at the same time learn about the specificity of interactions at each life stage of the parasite [20,61].

Our results come with limitations. Because of a small sample size, high biological variation within a group and small fold changes associated with the use of whole brain sampling, using an FDR resulted in little or no significant differentially expressed genes depending on the comparison. Genes found to be up- or downregulated in infected fish will therefore each necessitate further functional validations, as for all gene expression studies that show an association between a phenotype and expression changes (rather than a causal link). Our test of a causal link between a disruption of the inositol pathway and behaviours typical of infected sticklebacks using a pharmacological treatment supports the notion that some of the genes differentially expressed in the brain of infected individuals are indeed associated with behaviour modification following infection. However, it is crucial to underscore that it is highly probable that most changes in gene expression do not affect behaviour. Some of them may control other changes observed in infected individuals at the physiological level, others may be related to a general host response to infection, while some of the changes in gene expression may be a side-product of the presence of the parasite, or simply be false positive. Once we confirm the causal role of a molecular pathway in behaviour variation, the next step would be to test which one, if any, of these pathways are directly manipulated by the parasite. In this host-parasite system, a next step would be to determine if the increase in impa1 activity is a direct manipulation by the parasite, an indirect effect of its presence or an active response from the host [19].

\section{(b) Overlap of exposed and infected fish transcriptomes (i) Overlap between exposed and infected fish}

Contrary to our prediction, infected and exposed fish did not have similar brain expression profiles. Only nine genes were differentially expressed both in exposed and infected fish compared to controls (electronic supplementary material, table S3). Interestingly, fold changes for these nine genes were very similar in amplitude between infected and exposed fish and were all in the same direction (see the electronic supplementary material, figure S2). Four of those nine genes were found as differentially expressed only in the comparison of each of these two treatments compared to control individuals: a solute carrier family protein, a myosin light chain, a lipase gastric and a spermine acetyltransferase. On the other hand, only one gene was significantly differentially expressed between infected fish and exposed fish: $g d p d 5 a$, a glycerophosphodiester phosphodiesterase domain containing 5a, which was downregulated in infected fish (fold change $=-0.418$, $p=0.002$ ). This gene codes for a protein proposed (by similarity) to be involved in neurite formation, the regulation of the metabolite glycerophosphocholine (which can act as an osmolyte, https://hmdb.ca/metabolites/HMDB0000086, [62]), and in the cleavage of the GPI anchor of reversion-inducing-cysteine-rich protein with kazel motifs (RECK), which in turns is involved in Wnt7-specific actions in the brain (https:// www.uniprot.org/uniprot/Q8WTR4,[63]). Interestingly, a genomic study on S. solidus [64] has shown the existence of mimicry proteins (similar to the vertebrate host protein), with one of them belonging to the Wnt protein family and being the same protein found to be over-expressed in the head of orthopterans infected by a behaviour-altering hairworm [65]. Based on these similarities between two manipulating parasites and the observed change in the host, it could be proposed that a general disruption of cell-to-cell communication leading to various changes in behaviour may be at play [66].

\section{(ii) Exposed fish also have a distinct gene expression profile}

Interestingly, the brains of exposed fish are also very different from the ones of healthy control fish. One hundred and fifty three genes were differentially expressed in exposed fish compared to control individuals (electronic supplementary material, table S4): 48 up- and 105 downregulated, with a median fold change of 0.34 (range: $0.22-1.43$ ) and -0.47 (range: -1.16 to -0.22 ), respectively. More than $85 \%$ of these genes were specific to that exposed versus control comparison, thus forming an 'exposed' genomic signature, including 95 of the 105 downregulated genes. The enrichment analysis performed on the exposed-specific genes showed no significant enrichment. Whether this unique expression profile of exposed fish is a cause of the failure of the parasite to successfully infect these fish and was already present before infection, or if it is a long-lasting consequence of exposure (or both) is unknown. Indeed, we cannot exclude the fact that individuals which became infected and the ones that did not were already different before the experimental infection, as interaction between the genotype of the host and the parasite has been previously shown [67]. However, this is unlikely in our case, as all individuals come from the same crosses and laboratory environment. One way to test that these differences in expression existed before exposure and are the cause of the resistance to the parasite would be to redo a brain transcriptome analysis on a much higher number of control sticklebacks to detect difference in expression in the genes assigned to the exposed genomic signature in the present study. If so, it would suggest that the genotype of a proportion of individuals is the cause of differential gene expression rather than exposure to the parasite. On the other hand, being exposed is known to modify the immune system of the host. Sticklebacks resistant to parasitic eye flukes have a higher basic immunocompetence than more susceptible hosts [68]. A transcriptomic study of changes in gene expression in the head kidney following exposure to $S$. solidus in sticklebacks found that ROS production and recycling, B-cell activation and targeting, and fibrosis appear to play important roles in defence against cestodes [32], but did not find significant differences in gene expression between exposed and control fish. Similarly, exposed but non-infected gilthead sea bream (Sparus aurata) appear closer to the unexposed fish than the infected fish in their gene expression [69].

It is worth noting that while successful infection resulted in the upregulation of genes in the brain, exposure without infection resulted mostly in lower expression of genes in the brain, suggesting that they reflect different processes. The transcriptome response to pathogen lines of different virulence also show this opposite response in a Daphnia host, with the infective line resulting in more downregulated genes, while the non-infective strain resulted in upregulation of genes, with little overlap between the genes affected [70]. It thus appears that exposure could have a significant and distinct effect in 
the brain of the host, even when infection ultimately fails, and that this effect is carried over several weeks.

\section{(c) Overlap of selective serotonin reuptake inhibitor- treated and infected fish transcriptomes}

The analysis of the overlap between SSRI and infected fish brain expression profiles revealed only four genes in common (electronic supplementary material, table S5). These four genes are related to cellular organization and transport or have unknown functions. In comparison, changes in gene expression specific to the SSRI treatment (electronic supplementary material, table S6) include genes that have functions related to neurotransmission. Drugs in the SSRI family are designed to target the serotonin axis, which has its own range of effects on behaviour and physiology. The small overlap between genes affected in infected individuals and in SSRI-treated ones may thus be explained in part by the fact that SSRIs are specifically designed for a unique target, while $S$. solidus affects several traits in addition to behaviour. Infections have other major impacts on host sticklebacks that include reduced body condition, changes in metabolism, nutrient balance and reproduction [7]. A small overlap does not suggest necessarily that $S$. solidus and SSRI target different molecular pathways, only that these similarities are masked by multiple molecular differences.

\section{Conclusion}

Parasitic alteration of animal behaviour is predicted to be caused by, and to result in, multiple physiological changes in the host. Our study aimed at determining the brain gene expression profiles of parasitized fish to identify a genomic signature that distinguishes a S. solidus-infected stickleback from a control fish. Obtaining a general portrait of molecular pathways affected in an infected host is a valuable step to help determine if these hosts are manipulated by their parasite. Indeed, once it is known what changes in the brain of a host, one can manipulate these molecular pathways to recreate the infected host phenotype (or parts of it) in healthy individuals and ultimately test evolutionary predictions about effects on the parasite's fitness of host's behavioural differences, as done in Gammarus [71]. Our results allow us to predict that if S. solidus directly manipulates its fish host, its manipulation factors could target the inositol pathway. A next step would thus be to determine if $S$. solidus directly alters the behaviour of its host by affecting the inositol pathway, and if it does, to test if sticklebacks with an experimentally altered inositol pathway consequently exhibit altered behaviours that increase the probability of being predated by the final avian host of S. solidus.

Ethics. The experimental exposure to S. solidus parasites and/or SSRIs was undertaken at the University of Leicester, UK, under the authority of a UK Home Office project license (PPL 70/8148, held by I.B.). The lithium exposure was performed at Université Laval under the CPAUL certificate number 2017085-2. The project was authorized by the Comité de Protection des Animaux de l'Université Laval (2014069-1).

Data accessibility. The RNA-seq dataset consists of raw read counts for the 17417 transcripts that passed quality control and filters. Behaviour data from the lithium treatment are presented for all individuals. Authors' contributions. L.G. designed the study with the input of N.A.-H. and I.B. L.G. extracted RNA from the brains. L.G. and F.O.H. performed transcriptomic and statistical analyses. V.A.A. designed and performed the lithium pharmacological manipulation experiments. L.G. and N.A.-H. drafted the manuscript with input from F.O.H., V.A.A., and I.B. All authors reviewed and gave input on the final version of the manuscript.

Competing interests. We declare we have no competing interests.

Funding. Funding for this project was provided by the Fonds de Recherche du Québec Nature et Technologie (FRQ-NT) through a Team project grant to N.A.-H., the Natural Sciences and Engineering Research Council of Canada Discovery grant to N.A.-H., and a NSERC Vanier fellowship to F.O.H.

Acknowledgements. We thank the IBIS genomics analysis platform for help with RNA extraction and library preparation, L. Benestan for the specific subsetting idea, and J. Turgeon, C. R. Landry and J. Le Luyer for discussion.

\section{References}

1. Poulin R. 2010 Parasite manipulation of host behavior: an update and frequently asked questions. Adv. Study Behav. 41, 151-186. (doi:10.1016/ S0065-3454(10)41005-0)

2. Seppälä 0, Karvonen A, Valtonen ET. 2006 Susceptibility of eye fluke-infected fish to predation by bird hosts. Parasitology 132, 575-579. (doi:10. 1017/S0031182005009431)

3. Lagrue C, Kaldonski N, Perrot-Minnot MJ, Motreuil S, Bollache L. 2007 Modification of hosts' behavior by a parasite: field evidence for adaptive manipulation. Ecology 88, 2839-2847. (doi:10. 1890/06-2105.1)

4. Poulin R. 1995 'Adaptive' changes in the behaviour of parasitized animals: a critical review. Int. J. Parasitol. 25, 1371-1383. (doi:10.1016/00207519(95)00100-X)

5. Smyth J. 1946 Studies on tapeworm physiology. J. Exp. Biol. 23, 47-70.
6. Clarke A. 1954 Studies on the life cycle of the pseudophyllidean cestode Schistocephalus solidus. Proc. Zool. Soc. Lond. 124, 257-302. (doi:10.1111/j. 1469-7998.1954.tb07782.x)

7. Barber I, Scharsack J. 2010 The three-spined stickleback-Schistocephalus solidus system: an experimental model for investigating host-parasite interactions in fish. Parasitology 137, 411-424. (doi:10.1017/S0031182009991466)

8. Meakins R, Walkey M. 1975 The effects of parasitism by the plerocercoid of Schistocephalus solidus Muller 1776 (Pseudophyllidea) on the respiration of the three-spined stickleback Gasterosteus aculeatus L. J. Fish Biol. 7, 817-824. (doi:10.1111/j.1095-8649.1975.tb04652.x)

9. Heins DC, Singer SS, Baker JA. 2000 Virulence of the cestode Schistocephalus solidus and reproduction in infected threespine stickleback, Gasterosteus aculeatus. Can. J. Zool. 77, 1967-1974. (doi:10.1139/z99-180)
10. Tierney J, Huntingford F, Crompton D. 1996 Body condition and reproductive status in sticklebacks exposed to a single wave of Schistocephalus solidus infection. J. Fish Biol. 49, 483-493.

11. Milinski M. 1985 Risk of predation of parasitized sticklebacks (Gasterosteus aculeatus L.) under competition for food. Behaviour 93, 203-216. (doi:10.1163/156853986X00883)

12. Giles N. 1987 Predation risk and reduced foraging activity in fish: experiments with parasitized and non-parasitized three-spined sticklebacks, Gasterosteus aculeatus L. J. Fish Biol. 31, 37-44. (doi:10.1111/j.1095-8649.1987.tb05212.x)

13. Tierney JF, Huntingford FA, Crompton DW. 1993 The relationship between infectivity of Schistocephalus solidus (Cestoda) and anti-predator behaviour of its intermediate host, the three-spined stickleback, Gasterosteus aculeatus. Anim. Behav. 46, 603-605. (doi:10.1006/anbe.1993.1229) 
14. Barber I, Huntingford FA, Crompton DWT. 1995 The effect of hunger and cestode parasitism on the shoaling decisions of small freshwater fish. J. Fish Biol. 47, 524-536. (doi:10.1111/j.1095-8649.1995. tb01919.x)

15. Grécias L, Hébert F0, Berger $(S$, Barber I, AubinHorth N. 2017 Can the behaviour of threespine stickleback parasitized with Schistocephalus solidus be replicated by manipulating host physiology? J. Exp. Biol. 220, 237-246. (doi:10.1242/jeb. 151456)

16. Demandt N, Saus B, Kurvers RH, Krause J, Kurtz J, Scharsack JP. 2018 Parasite-infected sticklebacks increase the risk-taking behaviour of uninfected group members. Proc. R. Soc. B 285, 20180956. (doi:10.1098/rspb.2018.0956)

17. Quinn T, Kendall N, Rich Jr H, Chasco B. 2012 Diel vertical movements, and effects of infection by the cestode Schistocephalus solidus on daytime proximity of three-spined sticklebacks Gasterosteus aculeatus to the surface of a large Alaskan lake. Oecologia 168, 43-51. (doi:10.1007/s00442-0112071-4)

18. Perrot-Minnot M-J, Sanchez-Thirion K, Cézilly F. 2014 Multidimensionality in host manipulation mimicked by serotonin injection. Proc. $R$. Soc. B 281, 20141915. (doi:10.1098/rspb. 2014.1915)

19. Herbison R, Lagrue C, Poulin R. 2018 The missing link in parasite manipulation of host behaviour. Parasites \& Vectors 11, 222. (doi:10.1186/s13071018-2805-9)

20. Hébert F0, Aubin-Horth N. 2014 Ecological genomics of host behavior manipulation by parasites. In ecological genomics: advances in experimental medicine and biology, vol. 781 (eds ( Landry, N Aubin-Horth), pp. 160-190. Dordrecht, The Netherlands: Springer.

21. Poulin R, Maure F. 2015 Host manipulation by parasites: a look back before moving forward. Trends Parasitol. 31, 563-570. (doi:10.1016/j.pt. 2015.07.002)

22. Øverli $\emptyset$, Pall $M$, Borg B, Jobling M, Winberg S. 2001 Effects of Schistocephalus solidus infection on brain monoaminergic activity in female threespined sticklebacks Gasterosteus aculeatus. Proc. R. Soc. B 268, 1411-1415. (doi:10.1098/rspb. 2001.1668)

23. Rubenstein DR, Hofmann HA. 2015 Proximate pathways underlying social behavior. Curr. Opin. Behav. Sci. 6, 154-159. (doi:10.1016/j.cobeha.2015. 11.007)

24. Macnab V, Scott AP, Katsiadaki I, Barber I. 2011 Variation in the reproductive potential of Schistocephalus infected male sticklebacks is associated with 11-ketotestosterone titre. Horm. Behav. 60, 371-379. (doi:10.1016/j.yhbeh.2011. 07.005)

25. LoBue CP, Bell MA. 1993 Phenotypic manipulation by the cestode parasite Schistocephalus solidus of its intermediate host, Gasterosteus aculeatus, the threespine stickleback. Am. Nat. 142, 725-735. (doi:10.1086/285568)
26. Scharsack JP, Kalbe M, Derner R, Kurtz J, Milinski M. 2004 Modulation of granulocyte responses in threespined sticklebacks Gasterosteus aculeatus infected with the tapeworm Schistocephalus solidus. Dis. Aquat. Organ. 59, 141-150. (doi:10.3354/ da0059141)

27. Scharsack JP, Koch K, Hammerschmidt K. 2007 Who is in control of the stickleback immune system: interactions between Schistocephalus solidus and its specific vertebrate host. Proc. R. Soc. B 274, 3151-3158. (doi:10.1098/rspb.2007.1148)

28. McDonnell CM, Alaux C, Parrinello H, Desvignes J-P, Crauser D, Durbesson E, Beslay D, Le Conte Y. 2013 Ecto-and endoparasite induce similar chemical and brain neurogenomic responses in the honey bee (Apis mellifera). BMC Ecol. 13, 25. (doi:10.1186/ 1472-6785-13-25)

29. Aubin-Horth N, Renn SC. 2009 Genomic reaction norms: using integrative biology to understand molecular mechanisms of phenotypic plasticity. Mol. Ecol. 18, 3763-3780. (doi:10.1111/j.1365-294X. 2009.04313.x)

30. Thomas F, Poulin R, Brodeur J. 2010 Host manipulation by parasites: a multidimensional phenomenon. Oikos 119, 1217-1223. (doi:10.1111/ j.1600-0706.2009.18077.x)

31. Scharsack JP, Gossens A, Franke F, Kurtz J. 2013 Excretory products of the cestode, Schistocephalus solidus, modulate in vitro responses of leukocytes from its specific host, the three-spined stickleback (Gasterosteus aculeatus). Fish Shellfish Immunol. 35, 1779-1787. (doi:10.1016/j.fsi.2013.08.029)

32. Lohman BK, Steinel NC, Weber JN, Bolnick DI. 2017 Gene expression contributes to the recent evolution of host resistance in a model host parasite system. Front. Immunol. 8, 1071. (doi:10.3389/fimmu.2017. 01071)

33. Whitfield CW, Cziko AM, Robinson GE. 2003 Gene expression profiles in the brain predict behavior in individual honey bees. Science 302, 296-299. (doi:10.1126/science.1086807)

34. Aubin-Horth N, Letcher BH, Hofmann HA. 2009 Gene-expression signatures of Atlantic salmon's plastic life cycle. Gen. Comp. Endocrinol. 163 278-284. (doi:10.1016/j.ygcen.2009.04.021)

35. Segal E, Friedman N, Koller D, Regev A. 2004 A module map showing conditional activity of expression modules in cancer. Nat. Genet. 36, 1090-1098. (doi:10.1038/ng1434)

36. Barber I. 2013 Sticklebacks as model hosts in ecological and evolutionary parasitology. Trends Parasitol. 29, 556-566. (doi:10.1016/j.pt.2013.09.004)

37. Adamo SA. 1997 How parasites alter the behavior of their insect hosts. Parasites and Pathogens (ed. NE Beckage), pp. 231-245. Boston, MA: Springer.

38. Helluy S, Thomas F. 2003 Effects of Microphallus papillorobustus (Platyhelminthes: Trematoda) on serotonergic immunoreactivity and neuronal architecture in the brain of Gammarus insensibilis (Crustacea: Amphipoda). Proc. R. Soc. B 270, 563-568. (doi:10.1098/rspb.2002.2264)

39. Tain L, Perrot-Minnot M-J, Cézilly F. 2006 Altered host behaviour and brain serotonergic activity caused by acanthocephalans: evidence for specificity. Proc. R. Soc. B 273, 3039-3045. (doi:10. 1098/rspb.2006.3618)

40. Shaw J, Korzan W, Carpenter R, Kuris A, Lafferty K, Summers C, Øverli $\emptyset$. 2009 Parasite manipulation of brain monoamines in California killifish (Fundulus parvipinnis) by the trematode Euhaplorchis californiensis. Proc. R. Soc. B 276, 1137-1146. (doi:10.1098/rspb.2008.1597)

41. Robinson MD, McCarthy DJ, Smyth GK. 2010 edgeR: a Bioconductor package for differential expression analysis of digital gene expression data. Bioinformatics. 26, 139-140. (doi:10.1093/ bioinformatics/btp616)

42. Ritchie ME, Phipson B, Wu D, Hu Y, Law CW, Shi W, Smyth GK. 2015 limma powers differential expression analyses for RNA-sequencing and microarray studies. Nucleic Acids Res. 43, e47. (doi:10.1093/nar/gkv007)

43. Tang $H$, Klopfenstein D, Pedersen B, Flick P, Sato $K$, Ramirez F, Yunes J, Mungall C. 2015 GOATOOLS: tools for gene ontology. Zenodo. See http://doi.org/ 10.5281/zenod0.31628.

44. Berridge MJ, Irvine RF. 1989 Inositol phosphates and cell signalling. Nature 341, 197-205. (doi:10. 1038/341197a0)

45. Frej AD, Otto GP, Williams RS. 2017 Tipping the scales: lessons from simple model systems on inositol imbalance in neurological disorders. Eur. J. Cell Biol. 96, 154-163. (doi:10.1016/ j.ejcb.2017.01.007)

46. Davanzo P, Thomas MA, Yue K, Oshiro T, Belin T, Strober M, McCracken J. 2001 Decreased anterior cingulate myo-inositol/creatine spectroscopy resonance with lithium treatment in children with bipolar disorder. Neuropsychopharmacology 24, 359-369. (doi:10.1016/50893-133X(00) 00207-4)

47. Goodwin FK, Jamison KR. 2007 Manic-depressive illness: bipolar disorders and recurrent depression. Oxford, UK: Oxford University Press.

48. Ferruz N, Tresadern G, Pineda-Lucena A, De Fabritiis G. 2016 Multibody cofactor and substrate molecular recognition in the myo-inositol monophosphatase enzyme. Sci. Rep. 6, 30275. (doi:10.1038/srep30275)

49. Beaulieu J-M, Caron MG. 2008 Looking at lithium: molecular moods and complex behaviour. Mol. Interv. 8, 230. (doi:10.1124/mi.8.5.8)

50. Schildkraut JJ, Kety SS. 1967 Biogenic amines and emotion. Science 156, 21-30. (doi:10.1126/science. 156.3771.21)

51. Saule $P$, Adriaenssens $E$, Delacre $M$, Chassande 0 , Bossu M, Auriault C, Wolowczuk I. 2002 Early variations of host thyroxine and interleukin-7 favor Schistosoma mansoni development. J. Parasitol. 88, 849-855. (doi:10.1645/0022-3395(2002)088[0849: EVOHTA]2.0.(0;2)

52. Iwata M, Yamanome T, Tagawa M, Ida H, Hirano T. 1989 Effects of thyroid hormones on phototaxis of chum and coho salmon juveniles. Aquaculture 82, 329-338. (doi:10.1016/0044-8486(89)90419-5)

53. Iwata M. 1995 Downstream migratory behavior of salmonids and its relationship with cortisol and 
thyroid hormones: a review. Aquaculture 135, 131-139. (doi:10.1016/0044-8486(95)01000-9)

54. Fisher SK, Heacock AM, Agranoff BW. 1992 Inositol lipids and signal transduction in the nervous system: an update. J. Neurochem. 58, 18-38. (doi:10.1111/j.1471-4159.1992.tb09273.x)

55. Webster J, Lamberton P, Donnelly C, Torrey E. 2006 Parasites as causative agents of human affective disorders? The impact of anti-psychotic, mood-stabilizer and anti-parasite medication on Toxoplasma gondii's ability to alter host behaviour. Proc. R. Soc. B 273, 1023-1030. (doi:10.1098/sppb.2005.3413)

56. Berger CS, Aubin-Horth N. 2018 An eDNA-qPCR assay to detect the presence of the parasite Schistocephalus solidus inside its threespine stickleback host. J. Exp. Biol. 221, jeb178137. (doi:10.1242/jeb.178137)

57. Chelikani P, Fita I, Loewen PC. 2004 Diversity of structures and properties among catalases. Cell. Mol. Life Sci. 61, 192-208. (doi:10.1007/s00018-0033206-5)

58. Schirmer R, Schöllhammer T, Eisenbrand $G$, KrauthSiegel R. 1987 Oxidative stress as a defense mechanism against parasitic infections. Free Rad. Res. Commun. 3, 3-12. (doi:10.3109/ 10715768709069763)

59. Rademacher T, Parekh R, Dwek R. 1988 Glycobiology. Annu. Rev. Biochem. 57, 785-838. (doi:10.1146/annurev.bi.57. 070188.004033)
60. Collins CM, Olstad K, Sterud E, Jones CS, Noble LR, Mo TA, Cunningham C0. 2007 Isolation of a novel fish thymidylate kinase gene, upregulated in Atlantic salmon (Salmo salar L.) following infection with the monogenean parasite Gyrodactylus salaris. Fish Shellfish Immunol. 23, 793-807. (doi:10.1016/j. fsi.2007.03.001)

61. Ponton $F$, Lefèvre $T$, Lebarbenchon $C$, Thomas $F$, Loxdale H, Marché L, Renault L, Perrot-Minnot MJ, Biron DG. 2006 Do distantly related parasites rely on the same proximate factors to alter the behaviour of their hosts? Proc. R. Soc. B 273, 2869-2877. (doi:10.1098/rspb.2006.3654)

62. Wishart DS et al. 2018 HMDB 4.0: the human metabolome database for 2018. Nucleic Acids Res. 46, D608-DD17. (doi:10.1093/nar/ gkx1089)

63. UniProt Consortium. 2019 UniProt: a worldwide hub of protein knowledge. Nucleic Acids Res. 47, D506-DD15. (doi:10.1093/nar/gky1049)

64. Hébert F0, Phelps L, Samonte I, Panchal M, Grambauer S, Barber I, Kalbe M, Landry CR, Aubin-Horth N. 2015 Identification of candidate mimicry proteins involved in parasite-driven phenotypic changes. Parasites \& Vectors 8, 1. (doi:10.1186/s13071-015-0834-1)

65. Biron DG, Marché L, Ponton F, Loxdale HD, Galéotti N, Renault L, Joly C, Thomas F. 2005 Behavioural manipulation in a grasshopper harbouring hairworm: a proteomics approach. Proc. R. Soc. $B$ 272, 2117-2126. (doi:10.1098/rspb.2005.3213)
66. Adamo SA. 2013 Parasites: evolution's neurobiologists. J. Exp. Biol. 216, 3-10. (doi:10. 1242/jeb.073601)

67. Barribeau SM, Sadd BM, du Plessis L, Schmid-Hempel P. 2014 Gene expression differences underlying genotype-by-genotype specificity in a host-parasite system. Proc. Natl Acad. Sci. USA 111, 3496-3501. (doi:10.1073/pnas. 1318628111)

68. Kalbe M, Kurtz J. 2006 Local differences in immunocompetence reflect resistance of sticklebacks against the eye fluke Diplostomum pseudospathaceum. Parasitology 132, 105-116. (doi:10.1017/S0031182005008681)

69. Piazzon MC, Estensoro I, Calduch-Giner JA, del Pozo R, Picard-Sánchez A, Pérez-Sánchez J, Sitjà-Bobadilla A. 2018 Hints on T cell responses in a fish-parasite model: Enteromyxum leei induces differential expression of $\mathrm{T}$ cell signature molecules depending on the organ and the infection status. Parasites \& Vectors 11, 443. (doi:10.1186/s13071-018-3007-1)

70. McTaggart SJ, Cézard T, Garbutt JS, Wilson PJ, Little TJ. 2015 Transcriptome profiling during a natural host-parasite interaction. BMC Genomics 16, 643. (doi:10.1186/s12864-015-1838-0)

71. Perrot-Minnot M-J, Cézilly F. 2013 Investigating candidate neuromodulatory systems underlying parasitic manipulation: concepts, limitations and prospects. J. Exp. Biol. 216, 134-141. (doi:10.1242/ jeb.074146)

\section{9}

